\title{
Urachal remnant carcinoma - a rare entity
}

\author{
V Naidu, ${ }^{1}$ MB ChB, FC Rad Diag (SA); N Maharaj, ${ }^{1}$ MB ChB, FC Rad Diag (SA); A Mitha, ${ }^{1}$ MB ChB, FC Rad Diag (SA); \\ J Maharajh, ${ }^{1}$ MB ChB, FF Rad (D) (SA), MMedRad (D); B Singh, ${ }^{2}$ MB ChB, FCS (SA), MD
}

${ }^{1}$ Department of Radiology, Nelson R Mandela School of Medicine, University of KwaZulu-Natal, Durban, South Africa

${ }^{2}$ Department of Surgery, Nelson R Mandela School of Medicine, University of KwaZulu-Natal, Durban, South Africa

Corresponding author: V Naidu (vanesha@mweb.co.za)

Primary malignancy of the urachal remnant is a rare neoplasm that accounts for less than $0.01 \%$ of all adult cancers, with an estimated annual incidence of 1:5 million. The tumour carries a grave prognosis that attests to its highly aggressive nature. Owing to its extra-peritoneal location, the tumour runs a relatively silent clinical course until late presentation, when most patients display extensive local invasion and metastatic spread. In this report, we highlight a case of primary malignancy of the urachus that on initial clinical evaluation masqueraded as a Sister Mary Joseph's nodule. Characteristic imaging features, however, proved decisive in establishing the diagnosis of a urachal carcinoma.

S Afr J Rad 2013;17(2):63-64. DOI:10.7196/SAJR.837

A 51-year-old man presented to the surgical department with bleeding from an ulcerated umbilical mass, associated with lower abdominal discomfort and painless haematuria. Abdominal examination revealed an irregular umbilical mass with central ulceration. A provisional clinical diagnosis of a Sister Mary Joseph's (SMJ) nodule was made. Blood results, abdominal X-rays and a barium enema study proved unremarkable in determining a primary lesion. Computed tomography (CT) of the chest and abdomen demonstrated an irregular soft-tissue mass extending from the urinary bladder dome to the umbilicus, with localised infiltration of the anterior abdominal wall and cutaneous surface (Fig. 1). The tumour displayed punctate calcification and heterogenous enhancement (Fig. 2). Bilateral pulmonary nodules were indicative of metastatic lung deposits. A voiding cystogram revealed an elongated 'teardrop' bladder with constant indentation and irregularity of the vesical dome suggestive of extrinsic compression with possible mural invasion (Fig. 3). A radiological diagnosis of a urachal malignancy was made. Magnetic resonance imaging (MRI) was not undertaken as it was thought to confer little benefit in the setting of advanced disease.

Following histological confirmation of moderately differentiated invasive adenocarcinoma of the urachal remnant, and administration of chemotherapy, the patient developed cerebral metastasis and died within a year of diagnosis.

\section{Discussion}

In early fetal development, the ventral cloaca (the precursor of the fetal bladder) is connected to the allantois via the urachus. The tubular urachus undergoes progressive involution, resulting in an obliterated, fibrous cord by 32 weeks' gestation. This vestigial remnant, also known as the median umbilical ligament, extends in the midline from the bladder apex to the umbilicus. It is located in the extraperitoneal space of Retzius, which is bounded by the parietal peritoneum posteriorly and the tranversalis fascia anteriorly. ${ }^{[1,2]}$ Incomplete regression of the urachus results in 4 recognisable anomalies, which are indicated in Fig. 4.

Acquired urachal conditions include infection and, rarely, malignancy. ${ }^{[1,2]}$ Malignant urachal neoplasms account for less than $0.5 \%$ of all bladder malignancies, affecting mainly men of $40-70$ years' age.
Although the urachus is normally lined by transitional epithelium, almost $90 \%$ of urachal tumours are adenocarcinomas, $75 \%$ of which are mucin producing. ${ }^{[1]}$ The pathophysiology is unclear; it has been proposed that metaplasia of the urachal mucosa into columnar epithelium followed by malignant transformation is the most probable aetiology. ${ }^{[3]}$

Patients with urachal carcinoma present with haematuria, dysuria, abdominal pain, umbilical mass or bloody, mucoid discharge. ${ }^{[4]}$ Urachal tumours arise in the juxtavesical portion of the urachus and extend inferiorly into the bladder and superiorly towards the umbilicus. ${ }^{[1]}$ Owing to its extraperitoneal location, the tumour has a silent clinical course. The late clinical presentation often delays appropriate therapeutic intervention; ${ }^{[5]}$ surgery is the mainstay, encompassing en bloc resection of the urachus, umbilicus, surrounding soft tissues, bilateral pelvic lymphadenectomy and partial/radical cystectomy. Radiotherapy and chemotherapy have shown no definitive success. ${ }^{[6]}$

Radiology plays an integral role in the diagnosis and staging of urachal malignancies. CT demonstrates a solitary midline mass located anterosuperiorly to the vesical dome, which may be solid, cystic or mixed. Heterogenous enhancement with intratumoral areas of low attenuation may be seen, representing pools of mucin or necrosis. Psammomatous calcification is a hallmark feature occuring in $50-70 \%$ of lesions. ${ }^{[4]}$ Stippled, punctate or curvilinear calcification is usually detected on the peripheral aspect of the tumour mass. CT is pivotal in tumour staging, demonstrating local extent of disease, pelvic lymph node involvement and systemic metastastic spread, commonly to lung, liver, brain or bone. Perilesional fat stranding is suggestive but not specific for malignancy as infection may display similar features. ${ }^{[1]}$ MRI confers similar findings to CT with regard to tumour location. The lesion is characteristically $\mathrm{T} 2$ hyperintense owing to the presence of intratumoural mucin. MRI also helps to determine the intravesical extent of tumour. ${ }^{[5]}$ On ultrasound, urachal carcinomas appear as cystic lesions with mixed echogenicity or as a solid mass. Areas of echogenicity are reflective of calcification or mucin. ${ }^{[4]}$

Haematuria is the most common presenting symptom in both urachal carcinoma and primary malignancy of the bladder. Considerable overlap in presentation makes it difficult to clinically differentiate between these two entities. It is imperative that optimal distinction be made as urachal 


\section{CASE REPORT}

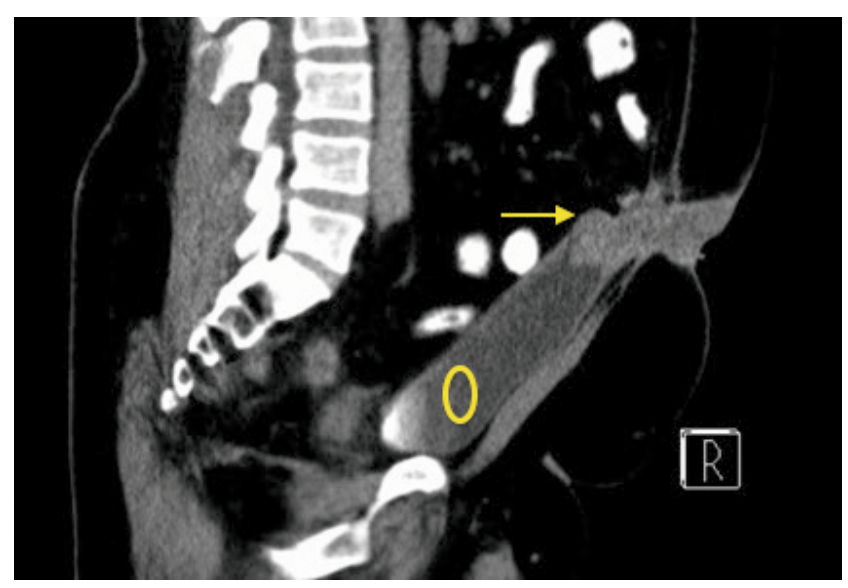

Fig. 1. Midline sagittal CT of the lower abdomen and pelvis demonstrates a heterogeneously enhancing soft tissue mass involving the bladder dome extending to the umbilicus with infiltration of the adjacent anterior abdominal wall (arrow). Note the elongated 'teardrop' bladder (circle).

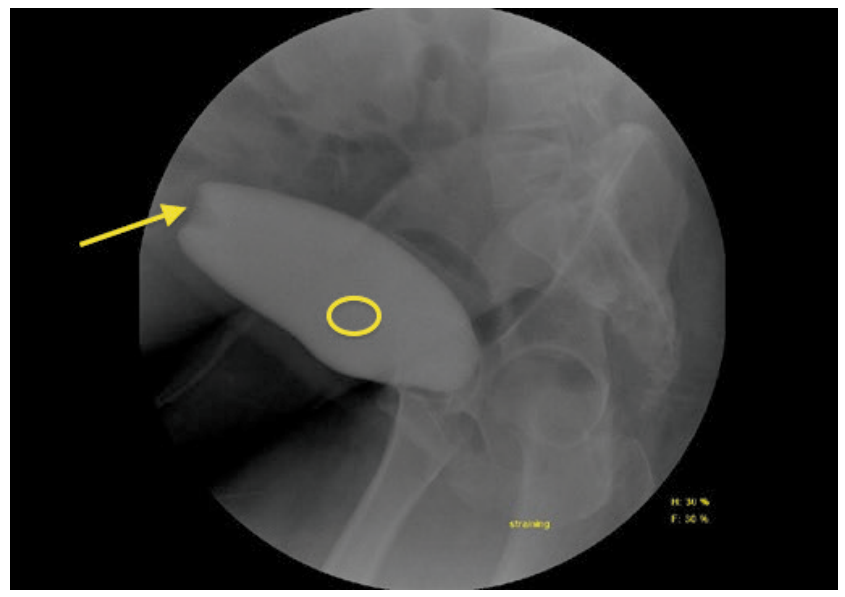

Fig. 3. Lateral cystogram view demonstrates irregular nodular indentation of the bladder dome (arrow) and elongated urinary bladder (circle).

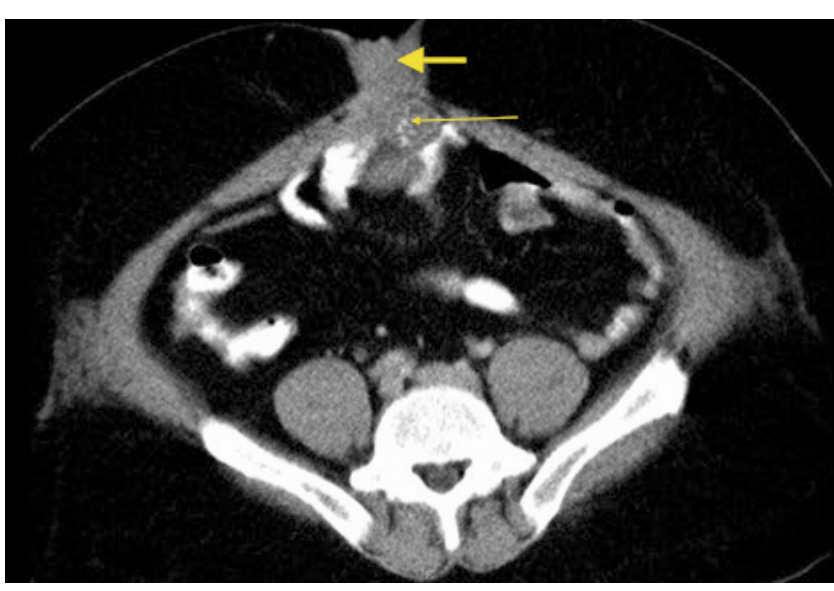

Fig. 2. Axial CT image through the superior aspect of the pelvis demonstrates a midline mass at the umbilicus (thick arrow) with fine punctate calcification (thin arrow).

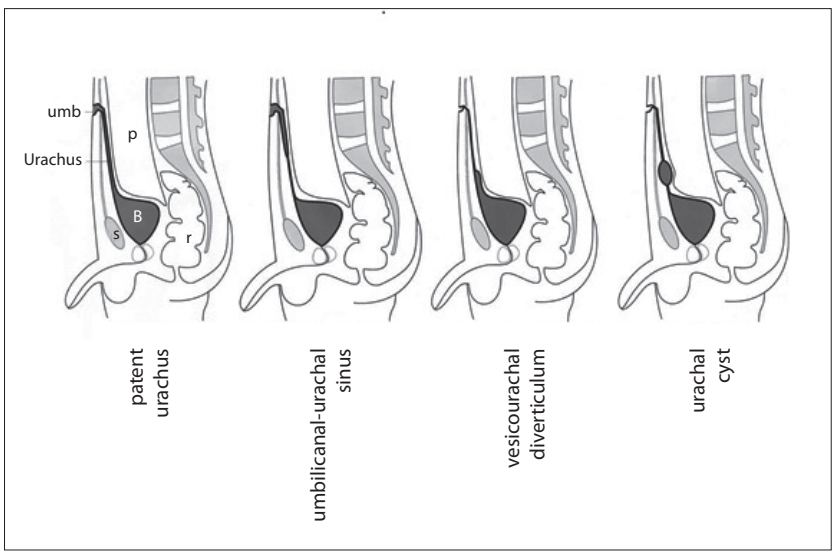

Fig. 4. Four types of congenital urachal anomaly. $B=$ bladder; $p=$ peritoneal cavity; $r$ = rectum; $s=$ symphysis; umb = umbilicus (by courtesy of Dr JeongSik $\left.Y u^{[l]}\right)$. carcinoma carries a worse prognosis and therapeutic management differs for both cancers. Even at cystoscopy, it is a clinical challenge to distinguish primary bladder tumour arising at the bladder apex from a urachal carcinoma. A tumour in the location of the bladder apex should alert the clinician to the possibility of a urachal malignancy and prompt further investigations. The characteristic imaging features and location of tumour assists accurate differentiation. Primary bladder carcinoma has a large intravesical component compared with urachal carcinomas that have a large extra-vesical component and a tendency to spread extraperitoneally into the space of Retzius and the umbilical region. ${ }^{[5]}$

Similarly to our reported case, urachal carcinoma can manifest as a mass in the region of the umbilicus. Clinically, it can closely resemble a SMJ nodule. Emphasis must be made that primary urachal carcinoma and a SMJ nodule are two very different pathologies. The eponymous nodule has been ascribed to umbilical or para-umbilical metastatic nodules that could be the first sign of malignancy from the gastrointestinal tract, the female genital tract or the genito-urinary tract. The radiological features of the nodule differ considerably from that of a primary urachal tumour, which is crucial in differentiating these umbilical entities. On imaging, a SMJ nodule appears as a discrete, welldefined mass confined to the subcutaneous tissues or intradermal layers of the anterior abdominal wall at the umbilical/periumbilical region. Unlike an urachal tumour, it does not demonstrate contiguity with the bladder dome or tumour extension along the urachal remnant.

\section{Conclusion}

Owing to its location and presentation, urachal carcinoma can clinically mimic a SMJ nodule. A sound understanding of the anatomy, a high index of clinical suspicion, and the radiological features of this tumour are crucial to an accurate diagnosis. As urachal carcinoma carries an unfavourable prognosis, early detection and accurate staging is imperative to expedite treatment and improve clinical outcome.

1. Yu JS, Kim KW, Lee HJ, Lee YJ, Yoon CS, Kim MJ. Urachal remnant diseases: spectrum of CT and US findings. Radiographics 2001;21(2):451-461.

2. Isaacs GS, Singh B. The micturating umbilicus. S Afr Med J 2010;100(1):22-23.

3. Nimmonrat A, Na-Chiang Mai W, Muttarak M. Urachal abnormalities: clinical and imaging features. Singapore Med J 2008; 49(11):930-935.

4. Mengiardi B, Weisner W, Stoffel F, et al. Case 44: adenocarcinomaof the urachus. Radiology 2002;222:744747.

5. Koster IM, Cleyndert P, Giard RW. Best cases from the AFIP: urachal carcinoma. Radiographics 2009; 29(3):939-942. [http//dx.doi.org/10.1148/rg.293085152]

6. Ashley RA, Inman BA, Sebo T], et al. Urachal carcinoma: clinicopathologic features and long-term outcomes of an aggressive malignancy. Cancer 2006;107(4):712-720. [http//dx.doi.org/10.1002/cncr.22060] 